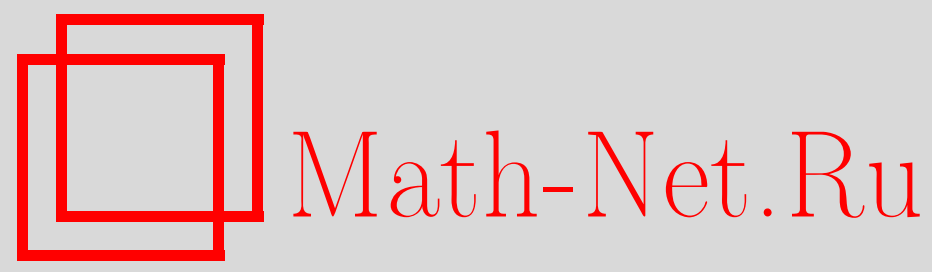

А. Н. Дегтев, Почти комбинаторно-селекторные множества, Матем. заметки, 2000, том 68, выпуск 6, 851-853

DOI: https://doi.org/10.4213/mzm1007

Использование Общероссийского математического портала Math-Net.Ru подразумевает, что вы прочитали и согласны с пользовательским соглашением http://www.mathnet.ru/rus/agreement

Параметры загрузки:

IP : 54.198 .55 .26

26 апреля 2023 г., $12: 24: 50$

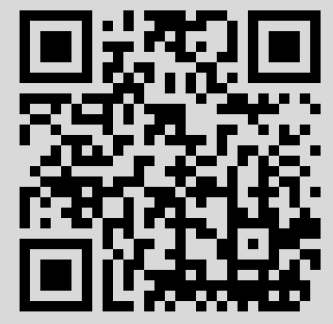


УДК 510.5

\section{ПОЧТИ КОМБИНАТОРНО-СЕЛЕКТОРНЫЕ МНОЖЕСТВА}

\section{А.Н. Дёгтев}

Исследуются классы подмножеств натурального ряда, основанные на понятии селекторных функций типа селектора полурекурсивного множества по Джокушу.

Библиография: 4 названия.

Пусть $\mathbb{N}=\{0,1,2, \ldots\}, A \subseteq \mathbb{N}$ и $\beta-n$-местная булева функция (Б $\Phi)$. Назовем $A$ почти $\beta$-комбинаторно-селекторным $(\beta$-КС) множеством, если найдутся элемент $\bar{a}$ из $\bar{A}=\mathbb{N} \backslash A$ и $n$-местная общерекурсивная функция (ОР $\Phi) f$ такие, что

$$
\begin{aligned}
& \left(\forall x_{1}, \ldots, x_{n}\right)\left(f\left(x_{1}, \ldots, x_{n}\right) \in\left\{x_{1}, \ldots, x_{n}, \bar{a}\right\}\right) \wedge \\
& \left.\quad\left(f\left(x_{1}, \ldots, x_{n}\right) \in A \Longleftrightarrow \beta\left(\chi\left(x_{1}\right), \ldots, \chi\left(x_{n}\right)\right)=1\right)\right),
\end{aligned}
$$

где $\chi$ - характеристическая функция множества $A$. В этом случае мы говорим, что $A$ является $\beta$-комбинаторно-селекторным множеством посредством ОРФ $f$. Цель данной работы - получить информацию о классах $K(\beta)$ всех почти $\beta$-КС множеств.

Если в $(*)$ убрать элемент $\bar{a}$, то мы придем к понятию $\beta$-селекторны $x$ множеств [1], классы которых для Б $\Phi$ таких, что $\beta(x, \ldots, x)=x$ (другие Б $\Phi$ неинтересны), совпадают или с классом $F^{(0)}$ всех рекурсивных множеств, или с классом $F^{(1)}$ всех полурекурсивных множеств, или с классом $F^{(\infty)}$ всех подмножеств $\mathbb{N}$. С другой стороны, в заметке [2] почти $x y$-КС множества были названы почти полурекурсивными. Такое название навеяно тем, что класс $x y$-селекторных множеств равен $F^{(1)}$ и равен классу $(x \vee y)$-селекторных множеств [3]. Ниже $\varnothing$ и $\mathbb{N}$ не считаются почти $\beta$-КС множествами и, в частности, они исключаются из $F^{(0)}$ и других классов. Далее, легко проверить, что $K(0)=F^{(\infty)}$ и если $\beta(0, \ldots, 0)=1$, то $K(\beta)=\varnothing$, откуда $K(1)=\varnothing$. Наконец, $K(x)=F^{(\infty)}$.

Лемма 1. Пусть $\beta(0, \ldots, 0)=0 u \beta$-немонотонная БФ. Тогда $K(\beta)=F^{(0)}$.

ДокАЗАТЕльСтво. Если $A \in F^{(0)}$ и $\bar{a} \in \bar{A}$, то положим по определению

$$
f\left(x_{1}, \ldots, x_{n}\right)= \begin{cases}\min \left\{x: x \in\left\{x_{1}, \ldots, x_{n}\right\} \cap A\right\}, & \text { если } \beta\left(\chi\left(x_{1}\right), \ldots, \chi\left(x_{n}\right)\right)=1 ; \\ \bar{a}, & \text { если } \beta\left(\chi\left(x_{1}\right), \ldots, \chi\left(x_{n}\right)\right)=0 .\end{cases}
$$

Ясно, что $A$ будет почти $\beta$-КС множеством посредством ОР $\Phi f$. Обратно, пусть функция $\beta=\beta\left(x_{1}, \ldots, x_{n}\right)$ немонотонная по некоторому аргументу, например, $x_{1}$. Тогда найдется набор $\theta_{2}, \ldots, \theta_{n} \in\{0,1\}$ такой, что $\beta\left(0, \theta_{2}, \ldots, \theta_{n}\right)=1$ и $\beta\left(1, \theta_{2}, \ldots, \theta_{n}\right)=0$. Отождествляем с $y$ те переменные $x_{i}$, для которьх $\theta_{i}=0$, и с $z$ те переменные $x_{j}$, для которых $\theta_{j}=1$. Если $A$ есть почти $\beta$-КС множество посредством ОР $\Phi f$, то сделаем 
в $f$ аналогичное отождествление переменных, получив ОР $\Phi g\left(x_{1}, y, z\right)$. Возьмем $a \in A$, и тогда очевидно, что $A=\{x: g(x, \bar{a}, a)=\bar{a}\}$. Значит, $A$ - рекурсивное множество.

Итак, интерес представляют лиш классы $K(\beta)$, где $\beta$-монотонные функции, отличные от констант, и $\beta \equiv x$.

ЛЕмма 2. Если БФ $\beta^{\prime}$ получена из $\beta$ в результате отоэлдествления некоторых из переменных или приписывания каким-то из них константы 0, то $K(\beta) \subseteq K\left(\beta^{\prime}\right)$.

ДокАЗАтЕЛЬСтво. Пусть $A$ - почти $\beta$-КС множество посредством ОР $\Phi f$. Если в $\beta$ отождествить $x_{1}$ с $x_{2}$ или приписать переменной $x_{1}$ константу 0 , получив БФ $\beta^{\prime}$, то $A$ окажется почти $\beta^{\prime}$-КС множеством посредством ОР $\Phi f^{\prime}$, которая получается из $f$ отождествлением в ней $x_{1}$ с $x_{2}$ или соответственно приписьванием переменной $x_{1}$ числа $\bar{a}$.

Обозначим через [ $\beta]$ наименьший замкнутый относительно суперпозиций класс Б $\Phi$, содержащий $\beta$.

Лемма 3. Если $\beta$ - монотонная Б $\Phi, \beta \neq 0,1, u \gamma \in[\beta]$, mо $K(\beta) \subseteq K(\gamma)$.

ДокАЗАТЕЛЬСТво. Пусть $\beta^{\prime}, \beta^{\prime \prime} \in[\beta]$ и

$$
\gamma\left(x_{1}, \ldots, x_{n-1}, y_{1}, \ldots, y_{m}\right)=\beta^{\prime}\left(x_{1}, \ldots, x_{n-1}, \beta^{\prime \prime}\left(y_{1}, \ldots, y_{m}\right)\right) .
$$

Нетрудно проверить, что если $A$ - почти $\beta$-КС и $\beta^{\prime \prime}-\mathrm{KC} \mathrm{множество} \mathrm{посредством} \mathrm{ОР \Phi} f^{\prime}$ и $f^{\prime \prime}$ соответственно, то $A$ будет и почти $\gamma$-КС множеством посредством ОР $\Phi$

$$
f^{\prime}\left(x_{1}, \ldots, x_{n-1}, f^{\prime \prime}\left(y_{1}, \ldots, y_{m}\right)\right) \text {. }
$$

Из описания всех замкнутых классов БФ [4] следует, что если $\beta$-монотонная БФ, $\beta \neq 0,1, x$, то имеет место один из следующих четырех случаев:

1) $x \vee y \in[\beta]$;

2) $x y \vee x z \vee y z \in[\beta]$

3) $x y \in[\beta]$;

4) $x y \in[\beta] \wedge \beta \notin[x y] \Longrightarrow x y \vee x z \in[\beta]$.

Отождествляя в Б $\Phi x y \vee x z$ переменную $z$ с переменной $y$, по лемме 2 получаем вложение $K(x y \vee x z) \subseteq K(x y)$.

ЛЕмма 4. Если $\beta$-монотонная Б $\Phi, \beta \neq 0,1$, то имеет место вложение

$$
K(x \vee y) \subseteq K(\beta)
$$

ДокАЗАТЕЛЬСТво. Если $A$ - почти $(x \vee y)$-КС множество посредством ОР $\Phi f$, то положим

$$
g(x, y)= \begin{cases}x, & \text { если } f(x, y) \in\{x, \bar{a}\} \\ y & \text { в противном случае. }\end{cases}
$$

Так как $g$ - селекторная ОРФ и

$$
(\forall x)(\forall y)(x \in A \vee y \in A \Longleftrightarrow g(x, y) \in A),
$$

то $A$ оказывается полурекурсивным множеством, т.е. $K(x \vee y)=F^{(1)}$. Но ОРФ $g$ позволяет по монотонной функции $\beta \neq 0,1, x$ определить ОР $\Phi$ такую, что [3] для всех $x_{1}, \ldots, x_{n} \in \mathbb{N}$ вьполнены следуюшие условия

1) $h\left(x_{1}, \ldots, x_{n}\right) \in\left\{x_{1}, \ldots, x_{n}\right\}$;

2) $\beta\left(\chi\left(x_{1}\right), \ldots, \chi\left(x_{n}\right)\right)=1 \Longleftrightarrow h\left(x_{1}, \ldots, x_{n}\right) \in A$.

Поэтому $K(x \vee y) \subseteq K(\beta)$. 
Лемма 5. Имеет место равенство $K(x \vee y)=K(x z \vee y z)$.

ДоКАЗАТЕльСтво. Если $A$ - почти $(x z \vee y z)-\mathrm{KC} \mathrm{множество} \mathrm{посредством} \mathrm{ОР} \Phi f$ и $a \in A$, то положим

$$
g(x, y)= \begin{cases}x, & \text { если } f(x, y, a) \in\{x, \bar{a}\} \\ y & \text { в противном случае. }\end{cases}
$$

Легко проверить, что $A$ посредством $\mathrm{OP} \Phi g$ является полурекурсивным множеством. Действительно, если $f(x, y, \bar{a})=\bar{a}$, то $x, y \notin A$. Если же $f(x, y, a)=x$ (соответственно $y$ ) и $x \neq \bar{a}$ (соответственно $y \neq \bar{a}$ ), то невозможно, что $x \notin A$ и $y \in A$ (соответственно $x \in A$ и $y \notin A)$. С другой стороны, как следует из леммы $4, K(x \vee y) \subseteq K(x z \vee y z)$.

Лемма 6. Имеет место равенство $K(x \vee y)=K(x y \vee x z \vee y z)$.

ДоКАЗАТЕЛЬСТво. Используя леммы 4 и 5 , достаточно показать, что

$$
K(x y \vee x z \vee y z) \subseteq K(x y \vee x z) .
$$

Пусть $A$ - почти $(x y \vee x z \vee y z)-\mathrm{KC}$ множество посредством ОР $\Phi f$. Положим

$$
g(x, y, z)=\left\{\begin{array}{l}
f(x, y, z), \text { если } f(x, y, z) \in\{x, \bar{a}\} ; \\
f(x, y, \bar{a}), \text { если } f(x, y, z)=y \text { и } y \notin\{x, \bar{a}\} \\
f(x, \bar{a}, z), \text { если } f(x, y, z)=z \text { и } z \notin\{x, \bar{a}\} .
\end{array}\right.
$$

Как и в доказательстве леммы 5 , нетрудно показать, что $A$ будет почти $(x y \vee x z)-\mathrm{KC}$ множеством посредством ОР $\Phi g$.

В статье [2] показано также, что существуют почти полурекурсивные, но не полурекурсивные множества и, разумеется, множества, не являюшиеся почти полурекурсивными. Отсюда и из лемм следует основная

ТЕорема. Если БФ $\beta$ такова, что $\beta(x, \ldots, x)=x$, то $K(\beta)$ совпадает с одним из классов

$$
F^{(0)} \subset F^{(1)} \subset K(x y) \subset F^{(\infty)},
$$

причем включения везде строгие.

\section{СПИСОК ЦИТИРОВАННОЙ ЛИТЕРАТУРЫ}

[1] Дёгтев А. Н. Рекурсивно-комбинаторные свойства подмножеств натуральных чисел // Алгебра и логика. 1990. Т. 29. №3. С. 303-314.

[2] Дёгтев А.Н. Почти полурекурсивные множества // Матем. заметки. 1999. Т. 66. № 2. C. $188-193$.

[3] Jockusch C. G. Semirecursive sets and positive reducibility // Trans. Amer. Math. Soc. 1968. V. 131. P. $420-436$.

[4] Яблонский С. В., Гаврилов Г. П., Кудрявцев В. Г. Функция алгебры логики и класса Поста. М.: Наука, 1966. 